\title{
BMJ Open Patient education materials for non- specific low back pain and sciatica: a protocol for a systematic review and meta-analysis
}

\author{
Bradley Furlong (D , ${ }^{1}$ Kris Aubrey-Bassler, ${ }^{1}$ Holly Etchegary, ${ }^{2}$ Andrea Pike, ${ }^{1}$ \\ Georgia Darmonkow, ${ }^{2}$ Michelle Swab, ${ }^{3}$ Amanda Hall ${ }^{1}$
}

To cite: Furlong B, AubreyBassler K, Etchegary $\mathrm{H}$, et al. Patient education materials for non-specific low back pain and sciatica: a protocol for a systematic review and meta-analysis. BMJ Open 2020;10:e039530. doi:10.1136/ bmjopen-2020-039530

- Prepublication history and additional material for this paper are available online. To view these files, please visit the journal online (http://dx.doi org/10.1136/bmjopen-2020039530).

Received 20 April 2020 Revised 11 June 2020 Accepted 01 July 2020

D Check for updates

(c) Author(s) (or their employer(s)) 2020. Re-use permitted under CC BY-NC. No commercial re-use. See rights and permissions. Published by BMJ.

${ }^{1}$ Primary Healthcare Research Unit, Memorial University of Newfoundland, St John's, Newfoundland, Canada ${ }^{2}$ Clinical Epidemiology, Memorial University of Newfoundland, St. John's, Newfoundland, Canada ${ }^{3}$ Health Sciences Library, Memorial University of Newfoundland, St. John's, Newfoundland, Canada

Correspondence to Bradley Furlong; bradley.furlong@mun.ca

\section{ABSTRACT}

Introduction Low back pain accounts for more disability than any other musculoskeletal condition and is associated with severe economic burden. Patients commonly present with negative beliefs about low back pain and this can have detrimental effects on their health outcomes. Providing evidence-based, patient-centred education that meets patient needs could help address these negative beliefs and alleviate the substantial low back pain burden. The primary aim of this review is to investigate the effectiveness of patient education materials on immediate process, clinical and health system outcomes.

Methods and analysis The search strategy was developed in collaboration with a librarian and systematic searches will be performed in MEDLINE, EMBASE, CINAHL, PsycINFO and SPORTDiscus. We will also search trial registries and grey literature through the OpenGrey database. Study selection will include a title and abstract scan and full-text review by two authors. Only randomised controlled trials will be included in this review. Trials must include patients with low back pain or sciatica and investigate educational interventions with at least one of the following contrasts: (1) education alone versus no intervention; (2) education alone versus another intervention; (3) education in addition to another intervention versus the same intervention with no education. Data extraction, risk of bias and grading of the quality of evidence will be performed independently by two reviewers. Risk of bias will be assessed using the PEDro scale, and the quality of evidence will be assessed with the Grades of Recommendation, Assessment, Development and Evaluation approach. A random-effects model will be used for each contrast, and results will be pooled if the participants, interventions, and outcomes are homogeneous. If heterogeneity is high $\left(\mathrm{I}^{2}>75 \%\right)$, we will evaluate the magnitude and direction of the differences in effect sizes across studies to determine if it remains reasonable to pool the results. Analyses of acute and subacute low back pain (less than 12 weeks duration) will be performed separately from chronic low back pain (12 weeks or greater duration). Likewise, analyses of shortterm (less than 6 months) and long-term (6 months or greater) follow-up will be performed separately. Subgroup analyses will be performed on non-specific low back pain, sciatica and mixed populations.
Strengths and limitations of this study

- Broad and comprehensive search strategy in several databases that will follow the Peer Review of Electronic Search Strategies guidelines, peer reviewed by two librarians.

- There will be no language restriction for relevant studies.

- This review will be limited to evidence from randomised controlled trials.

- Heterogeneity between interventions may prevent us from conducting a meta-analysis.

Ethics and dissemination Ethical approval is not required for this review. This study, along with its results, will be published in a peer-reviewed journal.

\section{INTRODUCTION}

Non-specific low back pain (LBP) is pain occurring below the rib cage and above the gluteal folds that is not due to a specific, identifiable cause. ${ }^{12}$ It is a very common condition from which many will recover within a few weeks; however, an estimated $23 \%$ of these patients tend to develop chronic LBP, defined as consistent LBP for 3 months or more, ${ }^{3}$ and up to $33 \%$ will likely experience a recurrence within a year. ${ }^{4}$ Recent data indicate that nonspecific LBP accounts for more disability than any other musculoskeletal condition ${ }^{5}$ and multiple studies show that the direct costs (eg, healthcare costs) and indirect costs (eg, industry productivity loss and compensation claims) ${ }^{6}$ associated with the disorder have a severe economic burden. ${ }^{78}$ Indeed, nonspecific LBP is one of the leading causes of work absenteeism ${ }^{8}$ and was associated with approximately 60.1 million years lived with disability in 2015. ${ }^{9} \mathrm{Katz}^{7}$ estimates the annual cost associated with the condition to be US $\$ 100-U S \$ 200$ billion in the USA alone. 
LBP is one of the five most common reasons why patients visit their family physicians. ${ }^{10}$ When visiting a doctor, most patients want information and reassurance about their LBP, ${ }^{11}$ but one study showed that participants were not satisfied with the information they received. ${ }^{12}$ Previous research indicates that patients may be dissatisfied because (1) providing satisfying information is especially difficult for non-specific LBP since patients cannot be presented with a specific diagnosis: ${ }^{13}$ (2) common treatments for non-specific LBP are not always effective; ${ }^{13}$ (3) health professionals have time constraints and may not always provide a detailed explanation of the condition $^{14}$ and (4) health professionals themselves may not be up to date with information about the condition and treatments. ${ }^{14}$ For these reasons, LBP care may become frustrating or confusing for patients, and may result in a spread of misinformation about LBP.

Though there are a limited amount of studies investigating the factors associated with negative beliefs about LBP, Bunzli et $a l^{5}$ found that these beliefs are associated with (1) patients' previous experience with pain, (2) diagnostic uncertainty, (3) being provided with a diagnosis of a condition that could not be fixed and (4) previous failed treatments. Negative beliefs are held despite the fact that non-specific LBP has a generally favourable prognosis ${ }^{16}$ and is considered to be self-limiting. ${ }^{17} \mathrm{~A}$ recent systematic review found that negative LBP beliefs are present in many populations and countries around the world. ${ }^{18}$ For example, Gross $e t a l^{19}$ found that most individuals in Canada hold pessimistic beliefs about LBP. They express concern about the severity and long term inevitably of LBP, and that it will most likely lead to disability in the future. Several studies show that negative patient beliefs about LBP, such as pain-related fear and pain catastrophising, are associated with LBP-related disability ${ }^{20}$ and may be more predictive of disability than pain intensity and duration. ${ }^{21}$ For example, fear-avoidance beliefs, pain catastrophising and beliefs/concern that non-specific LBP is a disabling condition are associated with low levels of physical activity and high levels of disability in patients with LBP. ${ }^{22}{ }^{23}$ Conversely, positive recovery expectations may lead to better outcomes ${ }^{24}$ and interventions aimed at reforming negative LBP beliefs into positive ones have been shown to improve LBP recovery. ${ }^{25} 26$

Patient education may be a helpful tool to increase satisfaction with care and mitigate the subsequent development of negative patient beliefs about LBP. Patient education involves providing advice and information to patients to help them better understand their condition(s). Doing so may help to modify negative beliefs that influence behaviour associated with the condition. ${ }^{27}$ LBP patient education aims to heighten patients' understanding of LBP, to reassure patients of the condition's favourable prognosis, and to provide patients with helpful tools to self-manage their LBP to reduce recurrence and healthcare dependency. ${ }^{13}$ Indeed, we know from a recent review by Lim $e t a l^{11}$ on the health information needs of people with LBP that patients want education-they want clear and consistent information about their LBP that is presented in language they can follow and include selfmanagement strategies and treatment options. Given this information, developing and implementing standardised, evidence-informed educational materials may, therefore, be a time and cost-efficient way of (1) providing patientcentred information that meets patient information needs; (2) addressing negative LBP beliefs by helping patients develop realistic expectations for their diagnosis and (3) relieving the healthcare system's LBP burden by providing healthcare professionals with evidenceinformed tools that can be promptly provided to patients, and which also keep healthcare professionals up to date with current LBP information.

Engers $e t a l^{1}$ conducted one of the first reviews on patient education materials (eg, an information booklet, pamphlet, leaflet or video) for LBP in 2008 searching studies published up to 2006. They identified 10 studies that assessed education versus no intervention of which only four assessed pain, five assessed disability and six assessed return to work. This review only included a narrative synthesis of the results and the effect sizes were not reported across studies, making it difficult to interpret the overall effect of education. Since this review, there have been additional systematic reviews that have assessed some form of patient education. ${ }^{28-40}$ However, most of these reviews have investigated more intensive formats of education or skills training programmes (eg, multisession and multicomponent education programmes or self-management interventions), ${ }^{28} 30$ 32-37 or a specific delivery method of education (eg, verbal and communicative education strategies) ${ }^{29}{ }^{38}$ rather than the provision of education materials. Similarly, some reviews only focused on a specific education topic such as neurophysiological pain education. ${ }^{31}{ }^{38}$ There were three reviews that explored the effectiveness of patient education that included studies involving education materials for various outcomes for LBP. ${ }^{28} 3940$ The most recent of these reviews was conducted by Zahari $e t a l^{40}$ They investigated the effectiveness of patient education interventions that could range from an information booklet to a multisession education programme on pain, disability and quality of life in elderly people ( $>60$ years of age). While they found that these types of education interventions were moderately effective, this only provides us with an update for a specific portion of the population of interest and on only a subset of the outcomes we are interested in. In terms of outcomes, few reviews have investigated the effect of patient education materials on important process outcomes such as knowledge, skills, fearavoidance and self-efficacy. There are only two reviews to our knowledge that have focused on these outcomes and included studies that used patient education materials as an intervention. ${ }^{28}{ }^{39}$ Traeger $e t a l^{39}$ focused on the outcome of reassurance (defined as reducing fear and concern) and Ainpradub et $a l^{28}$ included fear-avoidance beliefs as an outcome. While Traeger $e t a l^{39}$ found positive effects on reassurance, Ainpradub et $a l^{28}$ found no effect 
on fear-avoidance beliefs. However, each of these reviews included different studies and both included interventions beyond the scope of patient education materials. Therefore, while there is currently a large breadth of evidence from available systematic reviews on patient education, none have focused specifically on the effectiveness of providing patient educational materials to patients on process, clinical and health system outcomes and thus the evidence remains out of date for this question.

Accordingly, the primary aim of this review is to provide up-to-date evidence on the effectiveness of patient education materials on immediate process outcomes such as knowledge, satisfaction and expectations; clinical outcomes such as pain and physical disability; and health system outcomes such as healthcare utilisation and costeffectiveness in patients with acute and chronic LBP.

\section{METHODS}

\section{Search strategy}

The search strategy will be adapted from the comprehensive search strategy developed by the Back Pain Cochrane review group for the review by Engers et al. ${ }^{1}$ This will be completed by an academic health sciences librarian with input from the project team, and will be peer reviewed by a second librarian following the Peer Review of Electronic Search Strategies guidelines. ${ }^{41}$ The following databases will be searched from inception to April 2020: MEDLINE, EMBASE, CINAHL, PsycINFO and SPORTDiscus. A draft of the adapted Ovid MEDLINE search strategy is presented in online supplementary appendix A. We will also search trial registries as well as grey literature through the OpenGrey database.

\section{Inclusion/exclusion criteria}

For this review, there will be no language restrictions. We will use Google translate for non-English studies. The remainder of the criteria are as follows:

\section{Study design}

Only randomised controlled trials (RCTs) will be included. Pilot and feasibility studies will be included so long as participants were randomly allocated to groups.

\section{Population}

Eligible studies will investigate adults aged 16 years or older with acute, subacute or chronic non-specific LBP or sciatica. Our definition of non-specific LBP will include populations with and without leg pain, but without nerve root compromise, as well as conditions such as spondylitis, spondylolysis, spondylolisthesis, disc protrusion, herniation or prolapse and radicular syndrome. Sciatica will be defined as pain radiating downwards from the buttock due to pressure on the lumbosacral nerve root. ${ }^{42}$ This nerve root compromise may involve inflammation or other immunological processes. ${ }^{43}$ Studies will be excluded if subjects have a specific pathology such as cauda equina syndrome, infection, neoplasm, fracture or inflammatory disease, or if a large portion of the included participants were pregnant or had spinal surgery in the previous 12 months as the patient education for these patients are likely to differ from patients with non-specific LBP.

\section{Interventions}

In terms of intervention, any study that investigates the effect of patient education will be included. Patient education will be defined as interventions in which there is a health encounter between a patient and physician (delivered in a one-to-one setting or in a groupbased medical appointment) in family practice and emergency department settings where information about LBP (eg, diagnosis, prognosis, self-management or other treatment advice) is provided to the patient by using a standardised evidence-based supplement. An evidence-based supplement can include structured pamphlets, booklets, links to online resources, audio files, videos or workbooks that are provided to the patient during or after consultation with the physician. Studies investigating education not delivered directly by a physician (eg, media campaigns), or education aimed solely at teaching subjects how to perform exercises will be excluded. Interventions in which the education provided to the patient is only provided verbally from the physician without an evidence-based supplement as described above will also be excluded. Education materials are often provided as one component in a larger multicomponent intervention; for this review, we are interested in interventions in which the educational material is the main component of the intervention. Therefore, interventions that include education, plus another conservative component such as physiotherapy which is considered to be the main component, will be excluded unless the comparison group allows us to isolate the effect of education.

\section{Comparison}

We will consider the effect of education compared with two main comparison groups (1) no other intervention and (2) another conservative intervention. In cases where education is part of a multi-component intervention and is not the main component, they will be included if the effect of the education alone can be determined (ie, education + other conservative components vs the conservative components alone which allows for determining the additive effect of education). In cases where the comparison group is described as usual care but is not explicitly defined as to what this entails, we will assume it to be the absence of an active intervention and included in the first comparison group. For studies that have a usual care comparison group which is defined and does include other interventions such as seeking care from health professionals or exercise therapy, this study will be included in the second comparison. Comparisons of nonconservative treatments (eg, spinal cord stimulations or surgery) will be excluded. 


\section{Outcomes}

For this review, we are interested in assessing the effectiveness of education at three different levels. First, we are interested in the effect of education on process outcomes. These are the variables that are directly targeted by the education intervention and are thought to influence the clinical outcomes including knowledge, pain self-efficacy, reassurance, pain-related anxiety, depression, coping, expectations and treatment satisfaction (these are also referred to as potential mediators of effect). Second, we are interested in the effect of education on clinical outcomes relevant to patients with LBP including shortterm and long-term measures of pain, physical disability, return to work and quality of life. Third, we are interested in the effect of education on health system outcomes including healthcare utilisation and cost-effectiveness. Studies that evaluate any of these outcomes will be included in this systematic review.

\section{Study selection}

Titles and abstracts of studies found in the literature search will be downloaded and imported to EndNote. ${ }^{44}$ Duplicates will be removed manually by the librarian and the resulting studies will be imported to Covidence systematic review software ${ }^{45}$ to perform the remainder of study selection. Titles and abstracts will be reviewed independently by two authors (BF and GD) for relevance, starting with a 10-study trial period to determine if a revision to the inclusion and exclusion criteria is required. Any conflicts will be discussed by the reviewers, and when necessary, a third reviewer will be consulted to resolve the conflict $(\mathrm{AH})$. The full texts of relevant studies will then be obtained, and full-text review will be performed by two independent reviewers (BF and GD). Conflicts will be discussed by the same reviewers and when necessary, a third reviewer to resolve the conflict $(\mathrm{AH})$. Reference lists of relevant studies will be hand searched to find studies missed by the search, and authors will be contacted to identify additional studies when conference abstracts or ongoing trials are found. If the full study of a conference abstract cannot be found it will be excluded.

\section{Data extraction}

Two reviewers will independently extract and chart the data of all included studies using standardised data extraction forms in Microsoft Excel (BF and GD). The extraction forms will include variables relating to study details (authors, year of publication, country of data collection), study characteristics (LBP type and duration, sample size, outcomes measures, study design, brief intervention group description, comparison group description). Intervention details will be extracted in accordance with the 12 variables outlined in the TIDieR checklist ${ }^{46}$ (eg, a description of the intervention procedures, who provided the intervention, how and where the intervention was provided, the frequency/dose and duration of the intervention, if and how adherence and fidelity were to be assessed, etc). Lastly, specific information on each outcome will be extracted including measurement tools, measurement scales, scoring methods and interpretation, mean, and standard deviation (SD). Point estimates of effect size and $95 \%$ CIs will be used to estimate the treatment effect. Review Manager V.5 will be used for the analysis.

After data extraction is complete, two authors will make independent judgements to include or exclude relevant studies for the meta-analysis. If all relevant data points are obtained, the study will be included.

\section{Risk of bias assessment}

Risk of bias will be assessed at the outcome level using the PEDro scale. ${ }^{47}$ The PEDro scale grades risk of bias on a 10-point scale. A study will be deemed to have a high risk of bias if 0-3 criteria on the scale are satisfied, moderate if 4-6 criteria are satisfied, and low if 7-10 criteria are satisfied. Two reviewers will independently assess risk of bias for all included studies (BF and GD). Conflicts will be discussed, and where necessary, will be resolved by a third reviewer (AH). Sensitivity analyses will be performed to determine if data from studies judged to have a high risk of bias influence the overall effect size.

\section{Data synthesis \\ Contrasts}

We are interested in assessing the effects of education in the following three scenarios:

1. Education alone versus no intervention.

2. Education alone versus another intervention.

3. Education in addition to another intervention versus the same intervention with no education.

\section{Effectiveness analysis}

As it is likely that different measurement tools will be used for each outcome, we plan to use the standardised mean difference for the analysis. A random-effects model will be used for each contrast since variation between each intervention is likely. We plan to pool the results if the participants, interventions and outcomes are homogeneous. We anticipate there will be a small degree of clinical heterogeneity in the types of educational materials (eg, content or delivery of the intervention) and populations assessed (eg, duration of LBP) for which we consider to be acceptable given our overall study question. If $\mathrm{I}^{2}>75 \%$, which represents potential for considerable statistical heterogeneity, we will investigate both the level of clinical heterogeneity as well as the magnitude and direction of the differences in effect sizes across studies to determine if it remains reasonable to pool the results. If heterogeneity is too high, or if there is only one study in the strata, we plan to develop a qualitative synthesis to describe the effect of the interventions. If meta-analyses are possible, we plan to perform subgroup analyses for hard copy (eg, booklets, pamphlets) and soft copy (eg, link to online resource, video) education material interventions. Subgroup analyses will also be performed for non-specific LBP, sciatica and mixed populations. A study will be considered to 
have a population of non-specific LBP if people with nerve root compromise are excluded. If there is no exclusion for nerve root compromise, then the population will be considered to be a mixed population. If only those with nerve root compromise are included in the study the population will be considered to be a sciatica population. Analyses of acute and subacute LBP (less than 12 weeks duration) will be performed separately from chronic LBP (12 weeks or greater duration). Likewise, analyses of shortterm (less than 6 months) and long-term (6 months or greater) follow-up will be performed separately. We also plan to perform a sensitivity analysis to determine if high risk of bias studies influence the results of the analysis.

To assess the level of certainty of the evidence, a summary of findings table will be developed for each outcome using the Grades of Recommendation, Assessment, Development and Evaluation (GRADE) approach. ${ }^{48}$ GRADE involves assessing each study using five domains, each of which are 'downgraded' a level of evidence if they meet the following criteria:

1. Quality-studies with high risk of bias contain greater than $25 \%$ of all participants.

2. Inconsistency-high heterogeneity is clear from visual inspection or $\mathrm{I}^{2}>75 \%$.

3. Indirectness-over $50 \%$ of participants are not in the target group (ie, if participants were subject to multicomponent interventions where the effect of education alone may not be interpretable).

4. Imprecision-the comparison for continuous data involves less than 400 participants, or there are less than 300 events for dichotomous data.

5. Publication bias-(A) many included studies have a small sample size, (B) studies are or are likely to be industry sponsored, or (C) other conflicts of interest are present. Publication bias will also be assessed from visual inspection of a funnel plot. The treatment effect from each study will be plotted against the sample size of each study. If the plot does not resemble a cone, or if the regression line is not perpendicular to the $\mathrm{x}$ axis then there may be publication bias. If any of these criteria are present, we will consider downgrading the quality of evidence of studies.

These will be assessed independently by two reviewers (BF and GD). Conflicts will be discussed, and if necessary, will be reviewed with a third author to come to a consensus (AH). Studies will be considered to have highquality evidence, moderate-quality evidence, low-quality evidence, very low-quality evidence or no evidence if there are zero to four downgrades, respectively.

\section{Dealing with missing data}

Authors will be contacted if data are missing from a study. Otherwise, the data will be obtained from graphs or calculated using other data in the study where possible. If a mean value cannot be obtained, the study will not be included in the meta-analysis, but instead used for descriptive review. If an SD is not provided, it will be calculated or estimated using a relevant statistic provided in the study (eg, from confidence intervals, standard errors, p values) ${ }^{49}$ If the SD cannot be calculated in this way, it may be imputed by borrowing values from similar studies, as described in the Cochrane handbook. ${ }^{50}$

\section{Patient and public involvement}

Patients and members of the public were involved in identifying and prioritising this question as part of an 'improving the management of LBP' key stakeholder engagement session held at Memorial University. During that session, patient-identified outcomes were also recorded and informed the choice of outcomes for this review. Neither patients nor members of the public were involved in the development of the protocol. Patients will be consulted again to review and validate components of education interventions and outcomes identified through the review according to their lived experience. Finally, patients will be consulted to help translate key messages of the results for dissemination.

\section{Ethics and dissemination}

Ethical approval is not required for this review. This study, along with its results, will be published in a peer-reviewed journal and the results may be summarised and circulated in other formats as appropriate (eg, infographics or evidence briefs). We have decided to publish rather than preregister this protocol as publishing has the added benefit of receiving critical appraisal and gives us the ability to provide a more detailed description of the methods and background of the study.

Contributors $\mathrm{AH}$ and $\mathrm{BF}$ conceptualised and designed this systematic review and meta-analysis. BF and $\mathrm{AH}$ drafted the protocol. $\mathrm{BF}, \mathrm{AH}$ and $\mathrm{MS}$ developed the search strategy and conducted the search. AH, KA-B and HE provided feedback on the manuscript for both content and clarity. All authors reviewed and provided feedback on the methods and analysis as well as the manuscript. BF and GD will perform study selection and data extraction. AH is the guarantor of this review.

Funding This study is supported by the Office of Research and Graduate Studies (Medicine) at Memorial University of Newfoundland.

Competing interests None declared.

Patient consent for publication Not required.

Provenance and peer review Not commissioned; externally peer reviewed.

Open access This is an open access article distributed in accordance with the Creative Commons Attribution Non Commercial (CC BY-NC 4.0) license, which permits others to distribute, remix, adapt, build upon this work non-commercially, and license their derivative works on different terms, provided the original work is properly cited, appropriate credit is given, any changes made indicated, and the use is non-commercial. See: http://creativecommons.org/licenses/by-nc/4.0/.

ORCID iD

Bradley Furlong http://orcid.org/0000-0002-2013-7624

\section{REFERENCES}

1 Engers $A$, Jellema $P$, Wensing $M$, et al. Individual patient education for low back pain. Cochrane Database Syst Rev 2008:CD004057.

2 Machado LAC, Kamper SJ, Herbert RD, et al. Analgesic effects of treatments for non-specific low back pain: a meta-analysis of placebo-controlled randomized trials. Rheumatology 2009;48:520-7.

3 Airaksinen O, Brox JI, Cedraschi C, et al. Chapter 4. European guidelines for the management of chronic nonspecific low back pain. Eur Spine J 2006;15 Suppl 2:s192-300. 
4 da Silva T, Mills K, Brown BT, et al. Risk of recurrence of low back pain: a systematic review. J Orthop Sports Phys Ther 2017;47:305-13.

5 Hoy D, March L, Brooks $\mathrm{P}$, et al. The global burden of low back pain: estimates from the global burden of disease 2010 study. Ann Rheum Dis 2014;73:968-74.

6 Kent PM, Keating JL. The epidemiology of low back pain in primary care. Chiropr Osteopat 2005;13:13.

7 Katz JN. Lumbar disc disorders and low-back pain: socioeconomic factors and consequences. J Bone Joint Surg Am 2006;88 Suppl $2: 21-4$.

8 Lidgren L. The bone and joint decade 2000-2010. SciELO Public Health 2003.

9 Vos T, Allen C, Arora M, et al. Global, regional, and national incidence, prevalence, and years lived with disability for 310 diseases and injuries, 1990-2015: a systematic analysis for the global burden of disease study 2015. The Lancet 2016;388:1545-602.

10 St. Sauver JL, Warner DO, Yawn BP, et al. Why patients visit their doctors: assessing the most prevalent conditions in a defined American population. In: Mayo Clinic Proceedings. . Elsevier, 2013: 88. 56-67.

11 Lim YZ, Chou L, Au RT, et al. People with low back pain want clear consistent and personalised information on prognosis, treatment options and self-management strategies: a systematic review. $J$ Physiother 2019;65:124-35.

12 Briggs AM, Slater H, Bunzli S, et al. Consumers' experiences of back pain in rural Western Australia: access to information and services, and self-management behaviours. BMC Health Serv Res 2012:12:357.

13 Cherkin DC, Deyo RA, Street JH, et al. Pitfalls of patient education. limited success of a program for back pain in primary care. Spine 1996;21:345-55.

14 Coulter A, Entwistle V, Gilbert D. Sharing decisions with patients: is the information good enough? BMJ 1999;318:318-22.

15 Bunzli S, Smith A, Schütze R, et al. Beliefs underlying pain-related fear and how they evolve: a qualitative investigation in people with chronic back pain and high pain-related fear. BMJ Open 2015;5:e008847.

16 Hayden JA, Dunn KM, van der Windt DA, et al. What is the prognosis of back pain? Best Pract Res Clin Rheumatol 2010;24:167-79.

17 Nasser MJ. How to approach the problem of low back pain: an overview. J Family Community Med 2005:12:3.

18 Morton L, de Bruin M, Krajewska M, et al. Beliefs about back pain and pain management behaviours, and their associations in the general population: a systematic review. Eur J Pain 2019;23:15-30.

19 Gross DP, Ferrari R, Russell AS, et al. A population-based survey of back pain beliefs in Canada. Spine 2006;31:2142-5.

20 Peters ML, Vlaeyen JWS, Weber WEJ. The joint contribution of physical pathology, pain-related fear and catastrophizing to chronic back pain disability. Pain 2005;113:45-50.

21 Denison E, Asenlöf P, Lindberg P. Self-Efficacy, fear avoidance, and pain intensity as predictors of disability in subacute and chronic musculoskeletal pain patients in primary health care. Pain 2004;111:245-52.

22 Barbosa FM, Vieira Érica Brandão de Moraes, Garcia JBS. Beliefs and attitudes in patients with chronic low back pain. BrJP 2018;1:116-21.

23 Elfving B, Andersson T, Grooten WJA. Low levels of physical activity in back pain patients are associated with high levels of fear-avoidance beliefs and pain catastrophizing. Physiother Res Int 2007:12:14-24

24 Hayden JA, Wilson MN, Riley RD, et al. Individual recovery expectations and prognosis of outcomes in non-specific low back pain: prognostic factor review. Cochrane Database Syst Rev 2019;2019:CD011284.

25 Buchbinder R, Jolley D. Improvements in general practitioner beliefs and stated management of back pain persist 4.5 years after the cessation of a public health media campaign. Spine 2007;32:E156-62.

26 George SZ, Teyhen DS, Wu SS, et al. Psychosocial education improves low back pain beliefs: results from a cluster randomized clinical trial (NCT00373009) in a primary prevention setting. Eur Spine J 2009;18:1050-8.
27 Slater H, Briggs AM, Watkins K, et al. Translating evidence for low back pain management into a consumer-focussed resource for use in community pharmacies: a cluster-randomised controlled trial. PLoS One 2013;8:e71918.

28 Ainpradub K, Sitthipornvorakul E, Janwantanakul P, et al. Effect of education on non-specific neck and low back pain: a meta-analysis of randomized controlled trials. Man Ther 2016;22:31-41.

29 Barbari V, Storari L, Ciuro A, et al. Effectiveness of communicative and educative strategies in chronic low back pain patients: a systematic review. Patient Educ Couns 2020;103:908-29.

30 Brox Jl, Storheim K, Grotle M, et al. Systematic review of back schools, brief education, and fear-avoidance training for chronic low back pain. Spine J 2008;8:948-58.

31 Clarke CL, Ryan CG, Martin DJ. Pain neurophysiology education for the management of individuals with chronic low back pain: systematic review and meta-analysis. Man Ther 2011;16:544-9.

32 Du S, Hu L, Dong J, et al. Self-Management program for chronic low back pain: a systematic review and meta-analysis. Patient Educ Couns 2017;100:37-49.

33 Du S, Yuan C, Xiao X, et al. Self-Management programs for chronic musculoskeletal pain conditions: a systematic review and metaanalysis. Patient Educ Couns 2011;85:e299-310.

34 Nicholl BI, Sandal LF, Stochkendahl MJ, et al. Digital support interventions for the self-management of low back pain: a systematic review. J Med Internet Res 2017;19:e179.

35 Oliveira VC, Ferreira PH, Maher CG, et al. Effectiveness of selfmanagement of low back pain: systematic review with meta-analysis. Arthritis Care Res 2012;64:1739-48.

36 Parreira P, Heymans MW, van Tulder MW, et al. Back schools for chronic non-specific low back pain. Cochrane Database Syst Rev 2017;8:CD011674.

37 Straube S, Harden M, Schröder H, et al. Back schools for the treatment of chronic low back pain: possibility of benefit but no Convincing evidence after 47 years of research-systematic review and meta-analysis. Pain 2016;157:2160.

38 Tegner H, Frederiksen P, Esbensen BA, et al. Neurophysiological pain education for patients with chronic low back pain. Clin J Pain 2018;34:778-86.

39 Traeger AC, Hübscher M, Henschke N, et al. Effect of primary care-based education on reassurance in patients with acute low back pain: systematic review and meta-analysis. JAMA Intern Med 2015;175:733-43.

40 Zahari Z, Ishak A, Justine M. The effectiveness of patient education in improving pain, disability and quality of life among older people with low back pain: a systematic review. J Back Musculoskelet Rehabil2019:1-10.

41 McGowan J, Sampson M, Salzwedel DM, et al. PRESS Peer Review of Electronic Search Strategies: 2015 Guideline Statement. J Clin Epidemiol 2016;75:40-6.

42 Ostelo RW. Physiotherapy management of sciatica. J Physiother 2020.

43 Stafford MA, Peng P, Hill DA. Sciatica: a review of history, epidemiology, pathogenesis, and the role of epidural steroid injection in management. Br J Anaesth 2007;99:461-73.

44 EndNote. Clarivate analytics PLC, 2020. Available: https://endnote. $\mathrm{com} /$

45 Innovation VH. Covidence systematic review software. Melbourne: Veritas Health Innovation VIC, 2016.

46 Hoffmann TC, Glasziou PP, Boutron I, et al. Better reporting of interventions: template for intervention description and replication (TIDieR) checklist and guide. BMJ 2014;348:91687.

47 Armijo-Olivo S, da Costa BR, Cummings GG, et al. PEDro or Cochrane to assess the quality of clinical trials? A metaepidemiological study. PLoS One 2015;10:e0132634.

48 Guyatt G, Oxman AD, Akl EA, et al. Grade guidelines: 1. IntroductionGRADE evidence profiles and summary of findings tables. $J$ Clin Epidemiol 2011;64:383-94.

49 Higgins JP, Deeks JJ. Chapter 7: selecting studies and collecting data. In: Cochrane Handb Syst Rev Interv. Version 510, 2008. www. handbook.cochrane.org

50 Higgins JP, Deeks JJ, Altman DG. Chapter 16: special topics in statistics. In: Cochrane Handb Syst Rev Interv. Version 510, 2011 www.handbook.cochrane.org 